m/s

médecine/sciences 1998 ; 14 : 259-61

\title{
PROTÉINE PHOSPHATASES : L'AUTRE PLATEAU DE LA BALANCE
}

\section{Hervé Chneiweiss}

\section{ADRESSE}

H. Chneiweiss : directeur de recherche au Cnrs, neurologue. Inserm U. 114, Collège de France, 11, place Marcellin-Berthelot, $75231 \mathrm{~Pa}-$ ris Cedex 05, France. es équilibres dynamiques caractérisent la biologie. Compte tenu du rôle incontournable des processus de phosphorylation dans la plupart des phénomènes de signalisation intracellulaire, sa contrepartie, c'est-àdire la déphosphorylation des protéines, joue un rôle tout aussi crucial. Mais un classique engouement premier pour les phénomènes qui paraissent positifs a longtemps privilégié l'étude des kinases, tandis que l'analyse des phosphatases s'est développée plus récemment. Cette étude a été permise, en particulier, grâce à la découverte d'inhibiteurs spécifiques, tels que les toxines d'éponge (l'acide okadaïque), ou d'algues bleues (la microcystine), ou encore d'extraits de cuticule de scarabées, comme la cantharidine connue depuis l'antiquité pour ses vertus aphrodisiaques.

Contrairement aux kinases eucaryotes, dont le nombre est évalué à près de 2000 chez les mammifères et dont la structure est remarquablement homogène, les phosphatases (un millier selon les calculs extrapolés des séquençages en cours) sont très diverses, et regroupées en trois familles géniques distinctes $[1,2]$. Deux de ces familles, PPP et PPM, représentant environ la moitié des phosphatases, déphosphorylent des phosphosérines ou des phosphothréonines. L'autre moitié déphosphoryle des phosphotyrosines [1,2].

\section{Les sérine/thréonine- phosphatases}

Les sérine/thréonine-phosphatases les plus abondantes appartiennent à la famille PPP, comme les classiques PP1, PP2A et PP2B (calcineurine), tandis que la famille PPM comprend la PP2C (dépendante du $\mathrm{Mg}^{2+}$ ) et la pyruvate déshydrogénase phosphatase mitochondriale. Les phosphatases PPP et PPM sont des familles de métalloenzymes contenant deux ions métalliques au site actif (fer et zinc pour $\mathrm{PP} 2 \mathrm{~B}$, fer et manganèse pour PP1, deux ions manganèse pour PP2C), catalysant la déphosphorylation par hydrolyse en une seule étape. L'architecture du domaine catalytique, telle qu'elle nous est révélée par la cristallographie par rayons X, est remarquablement conservée [3]. En outre, les séquences d'une même phosphatase ont été très fortement conservées au cours de l'évolution, permettant d'identifier aisément une phosphatase homologue chez la levure, la drosophile ou les mammifères, et autorisant des expériences de complémentations entre organismes très distants. Notre connaissance de la diversité des phosphatases a ainsi été grandement facilitée par les programmes de séquençage du génome de la levure, et l'étude chez la levure des effets de mutations a déjà permis de mieux comprendre certaines de leurs fonctions.

La grande diversité moléculaire des phosphatases est créée par la combinatoire des domaines et des sous-unités régulatrices [2]. A titre d'exemple, et chez les mammifères, on connaît actuellement 3 gènes d'unités catalytiques et 15 gènes de sous-unités régulatrices pour $\mathrm{PP} 1,12$ gènes de sous-unités régulatrices pour PP2A. Ce sont les sous-unités régulatrices qui confèrent la spécificité de fonction, en déterminant la localisation cellulaire de l'enzyme, en modulant son activité et son affinité pour telle ou telle protéine phosphorylée. Au 
cours des dernières années, de nombreux inhibiteurs endogènes des phosphatases ont été caractérisés. De façon intéressante, il s'agit souvent de protéines elles-mêmes phosphorylées, multipliant ainsi les intrications de cascades de régulation et permettant un ajustement fin des phénomènes de phosphorylation et de déphosphorylation. Un exemple d'un tel inhibiteur est la DARPP-32 (dopamine and cyclic AMP-regulated phosphoprotein), enrichie dans le système nerveux central et inhibiteur de la PP1 lorsqu'elle est phosphorylée par la kinase dépendante de l'AMP cyclique [4]. La DARPP-32 est ellemême déphosphorylée par la calcineurine (PP2B). Élévation d'AMPc intracellulaire et augmentation de calcium libre exercent ainsi des rôles antagonistes sur l'activité de la PP1 via l'état de phosphorylation de la DARPP-32 .

De façon presque étonnante, seules deux formes de PP2B ont été identifiées. Autre particularité, une longue boucle carboxy-teminale exerce un effet auto-inhibiteur sur le domaine catalytique. L'interaction avec la sous-unité régulatrice, capable de lier le calcium ou le complexe calcium-calmoduline, lève l'inhibition et active l'enzyme. La phosphatase PP2B est très enrichie dans le cerveau, d'où son autre dénomination de calcineurine. Elle est également essentielle à l'activation lymphocytaire $\mathrm{T}$ en déphosphorylant le facteur de transcription NF-AT. Les immunosuppresseurs tels que la ciclosporine et le FK506, en se liant à leurs récepteurs endogènes, respectivement la cyclophiline et la FKBP-12, inhibent la déphosphorylation de NFAT par PP2B [5].

La phosphatase PP2C résume presque la famille PPM et semble particulièrement impliquée dans la régulation des déphosphorylations de la cascade de stress chez les procaryotes comme chez les eucaryotes. Par exemple, chez la plante Arabidopsis, PP2C est un effecteur intracellulaire de l'acide abscissique permettant le maintien des graines dans un état de dormance. Chez la levure, elle est activée en réponse à un choc osmotique ou à un choc thermique. Chez les mammifères, l'activation de PP2C cholestérol et des acides gras induite dans les hépatocytes par l'augmentation du rapport AMP/ATP.

\section{PPP et cycle cellulaire}

Récemment trois nouveaux groupes de phosphatases de la famille PPP ont été découverts : PP4, PP5 et PP6, qui paraissent agir au cours du cycle cellulaire [6]. La séquence de PP4 est extraordinairement conservée entre les mammifères et la drosophile ; l'identité de séquence est de l'ordre de $94 \%$, c'est-à-dire d'un niveau voisin de celui de PP1 et PP2A, jusqu'ici les enzymes connues pour être les mieux conservées au cours de l'évolution. PP4 est nucléaire et semble impliquée dans la nucléation des microtubules dans le centrosome, une fonction attribuée jusqu'alors à PP2A. En prélude à la mitose, le centrosome se dédouble et forme les pôles du futur fuseau mitotique. PP4 est fortement localisée à ce niveau tout au long de la mitose, sauf lors de la télophase. Lors de la sortie du cycle cellulaire et de l'entrée en interphase, PP4 se localise dans le centrosome des cellules filles. La phosphatase PP5 semble également jouer un rôle important dans le cycle cellulaire. Reconnue chez la levure, la drosophile et les mammifères, elle est caractérisée par la présence d'un domaine auto-inhibiteur présentant des répétitions de séquences de tétratricopeptides (TPR). En outre, PP5 est fortement activée in vitro par les acides gras polyinsaturés comme l'acide arachidonique, une stimulation induite par liaison de l'acide gras aux TPR. Dans le noyau, PP5 participe à la régulation de la trancription des ARN ribosomiques. Les résultats obtenus avec l'analogue de PP6 chez la levure indiquent que cette phosphatase est nécessaire au passage de G1 vers $S$ au cours du cycle cellulaire. La présence de PP6 permet l'accumulation d'ARNm de certaines cyclines, nécessaires également lors de la transition G2/M.

\section{Les tyrosine- phosphatases}

Sur la base de leurs fonctions, de leurs structures et de leurs séquences, les tyrosine-phosphatases ont été regroupées en 4 familles : les phosphatases spécifiques des tyrosines (PTP), VH1, CDC25 et les tyrosine-phosphatases légères. Comme dans le cas des sérine/thréoninephosphatases, les familles de tyrosinephosphatases partagent entre elles des analogies de séquences très limitées, mais possèdent en commun un motif caractéristique de leur site actif composé d'une cystéine et d'une arginine distantes de 5 résidus, le motif Cx5R [7]. Une mutation de la cystéine en sérine fait perdre toute activité à l'enzyme, tandis qu'une mutation sur l'arginine modifie seulement les paramètres cinétiques. Ces phosphatases ne requièrent ni ion ni co-facteur mais, outre la cystéine et l'arginine, il existe un acide aspartique participant nécessairement à la structure tridimensionnelle du site actif. Les tyrosine-phosphatases partagent également une inhibition par le vanadate. Si la phosphotyrosine est le résidu préférentiellement déphosphorylé, certaines de ces phosphatases sont également capables de déphosphoryler des phosphosérines ou des phosphothréonines, comme par exemple CDC25 (voir l'article de C. Cans et al., p. 269 de ce numéro) et VH1.

Les PTP sont les plus nombreuses et les mieux caractérisées, et plusieurs structures cristallographiques sont déjà disponibles. Deux sous-familles existent selon que la molécule est cytoplasmique ou transmembranaire, ressemblant alors à un récepteur qui paraît être impliqué dans des phénomènes de reconnaissance intercellulaire. C'est à la première sous-famille, dont plusieurs membres participent à la transmission intracellulaire des effets des facteurs de croissance, qu'est consacrée la revue de S. Olivero et al. (voir p. 262 de ce numéro). Également impliquées dans la croissance cellulaire, les MAP-kinases phosphatases, par exemple CL100, sont des phosphatases à double spécificité (pTyr et pSer/pThr) appartenant à la famille VH1. L'hydrolyse de la phosphotyrosine est toutefois 50 à 1000 fois plus rapide que celle de la phosphothréonine ou de la phosphosérine. 


\section{En perspective}

Le nombre et la diversité des phosphatases continuent de croître. Audelà de séquences primaires très disparates, les données de la cristallographie ont révélé d'étonnantes similitudes structurales. Il reste beaucoup à comprendre sur les fonctions spécifiques dans lesquelles elles sont engagées, et leur propre mode de régulation. Ces données fondamentales ouvrent de nouveaux horizons dans le champ clinique où l'inhibition de PP2B lymphocytaire par la ciclosporine ou le FK506 est déjà largement utilisée. Des données récentes montrent également l'implication de tyrosine-phosphatases bactériennes en pathologie infectieuse. Ainsi la PTP YopH détermine la virulence de Yersi- nia, bacille responsable de la peste, en déphosphorylant un petit nombre de substrats récemment identifiés de l'hôte (voir l'article de S. Olivero et al., p. 262 de ce numéro), et il existe une PTP homologue chez Salmonella

\section{RÉFÉRENCES}

1. Hunter T. Protein kinases and phosphatases : the Yin and Yang of protein phosphorylation and signaling. Cell 1995 ; 80 : 225-36.

2. Wera S, Hemmings BA. Serine/threonine phosphatases. Biochem J 1995 ; 311 : 17-29.

3. Barford D. Molecular mechanisms of the protein serine/threonine phophatases. Trends Biochem Sci 1996 ; 21 : 407-12.

4. Shenolikar S, Nairn AC. Protein phosphatases : recent progress. Adv Second Messenger Phosphoprotein Res 1991 ; 23 : 1-121.
5. Baumann G, Borel J. Mécanismes moléculaires de l'action des agents immunosuppresseurs. Med Sci 1992 ; 8 : 366-71.

6. Cohen PTW. Novel protein serine/threonine phosphatases : variety is the spice of life. Trends Biochem Sci 1997 ; 22 : 245-51.

7. Fauman EB, Saper MA. Structure and function of the protein tyrosine phosphatases. Trends Biochem Sci 1996 ; 21 : 413-7.

\section{Remerciements}

Je remercie Jean-Antoine Girault pour sa relecture attentive de ce manuscrit et ses commentaires critiques.

\section{TIRÉS À PART}

H. Chneiweiss.

\title{
Vle Journée ANNUElLE du Club DES NeUROSCIENCES de CRÉTEIL
}

\section{Sous l'égide de I'Université Paris XII-Val-de-Marne et de I'Institut Mondor de Médecine Moléculaire (IM3) avec le soutien de l'ANPP}

\section{Le Jeudi 28 mai 1998}

\author{
Faculté de Médecine de Créteil - Amphi 2
}

\section{GLIE - GLIOSE - GLIOME}

9 h 00 Introduction

Guy Atlan - Président de l'Institut Mondor de Médecine Moléculaire (IM3)

Modérateurs : Brigitte Onténiente, Pierre Cesaro

9 h 15 - 10 h 15 Jacques Glowinski (Inserm U.114/Collège de France, Paris)

Relations astrocyto-neuronales. Rôle de l'acide arachidonique

10 h 30 - 11 h 30 Michel Mallat (Inserm U.495, Pitié-Salpêtrière, Paris)

Croissance et réaction microgliale : mécanisme et aspect fonctionnel

11 h 30 - 12 h 30 Dionysia Theodosis (Inserm U.378, Bordeaux)

Plasticité des interactions neurone/glie

Modérateurs : Josette Cadusseau, Romain Ghérardi

14 h 00 - 15 h 00 Manuel Nieto-Sampedro (Institut Cajal - Madrid)

Inhibitions of neurite outgrowth in brain gliotic tissue

15 h 00 - 16 h 00 Anne Baron-Van Evercooren (Inserm CJF 96-08, Pitié-Salpêtrière, Paris)

Approches expérimentales de la remyélinisation du SNC

16 h 00 - 17 h 00 Catherine Daumas-Duport (Service d'Anatomie Pathologique, St Anne, Paris)

Gliomes. Étude anatomo-pathologique

Club des Neurosciences de Créteil, Inserm U.421, 8, rue du Général-Sarrail, 94010 Créteil Cedex, France Téléphone : 0149813682 - Télécopie : 0149813709 -E-mail : ribeil@im3.inserm.fr 